\title{
1 Cardiac Microlesions Form During Severe Bacteremic Enterococcus faecalis Infection
}

2 Armand O. Brown ${ }^{1}$, Kavindra V. Singh ${ }^{2}$, Melissa R. Cruz ${ }^{1}$, Karan Gautam Kaval ${ }^{1}$, Liezl E.

3 Francisco ${ }^{3}$, Barbara E. Murray ${ }^{1,2}$, and Danielle A. Garsin ${ }^{1 *}$

4

$5 \quad{ }^{1}$ Department of Microbiology and Molecular Genetics, The University of Texas Health Science

6 Center at Houston, Houston TX, USA

$7 \quad 2$ Division of Infectious Diseases, Department of Internal Medicine, The University of Texas Health

8 Science Center at Houston, Houston, TX, USA

$9{ }^{3}$ Department of Biochemistry and Structural Biology, The University of Texas Health Science

10 Center at San Antonio, San Antonio, TX, USA

11

$12{ }^{*}$ Corresponding author

13 Danielle.A.Garsin@uth.tmc.edu

14 713-500-5454

15

16

17

18 Running Title: Cardiac microlesions formed by E. faecalis 


\section{Abstract}

Enterococcus faecalis is a significant cause of hospital-acquired bacteremia. Herein, the

discovery is reported that cardiac microlesions form during severe bacteremic $E$. faecalis infection

in mice. The cardiac microlesions were identical in appearance to those formed by Streptococcus

pneumoniae during invasive pneumococcal disease (IPD). However, E. faecalis does not encode

the virulence determinants implicated in pneumococcal microlesion formation. Rather, disulfide

bond forming protein DsbA was found to be required for $E$. faecalis virulence in a $C$. elegans

model and was necessary for efficient cardiac microlesion formation. Furthermore, E. faecalis

promoted cardiomyocyte apoptotic and necroptotic cell death at sites of microlesion formation.

Additionally, loss of DsbA caused an increase in pro-inflammatory cytokines unlike the wild-type

strain, which suppressed the immune response. In conclusion, we establish that $E$. faecalis is

capable of forming cardiac microlesions and identify features of both the bacterium and the host

response that are mechanistically involved.

SUMMARY: This work presents the observation of cardiac microlesion formation during severe blood stream infection with Enterococcus faecalis in mice. Moreover, we identify the contribution of a novel enterococcal virulence determinant in modulating microlesion formation and the host immune response.

KEYWORDS: Enterococcus faecalis, cardiac, microlesions, sepsis, bacteremia, infection, virulence, thioredoxin, disulfide bond, necroptosis 
Introduction

Enterococcus faecalis is a Gram-positive bacterium that normally co-exists with the host as a harmless commensal. However, it is capable of causing a variety of infections such as infective endocarditis (IE), urinary tract infection (UTI), bacteremia, peritonitis, prosthetic joint infection, and endophthalmitis. A common complication associated with enterococcal bacteremia is endocarditis, an infection that has a 1-year mortality rate of approximately $29 \%$ despite antibiotic therapy [1]. Enterococci are the third most common cause of endocarditis in North America [2] and, in patients with this infection, heart failure has been observed to occur with increased frequency [3-9]. Endocarditis only involves infection of the surfaces of the heart - the formation

of biofilms on heart valves and inner chambers that develop into complex masses called vegetations [10]. E. faecalis invasion of the deeper myocardial tissue to cause cardiac microlesions has not previously been described.

Interestingly, another Gram-positive pathogen, Streptococcus pneumoniae, was recently shown to invade the heart tissue during invasive pneumococcal disease (IPD) [11]. Importantly, pneumococcal microlesions were determined to be associated with altered cardiac electrophysiology, heart failure and scar formation during convalescence, which is thought to predispose individuals to major adverse cardiac events (MACE) [11, 12]. Furthermore, cardiac microlesions form following vascular endothelial cell invasion into the myocardial tissue through the interactions of pneumococcal Choline binding protein $A(C b p A)$ and pneumococcal teichoic acid associated phosphorylcholine (ChoP) with host laminin receptor (LR) and platelet activating factor receptor (PAFr), respectively. Cardiomyocyte death is mediated by exposure to the pneumococcal secreted cholesterol-dependent pore forming toxin, pneumolysin [11, 12], and 
similar observations were seen during blood stream infections with Francisella tularensis [15], and Mycobacterium avium [16], however the mechanisms involved were not characterized.

71 In this report, we show that cardiac microlesions also form during invasive E. faecalis infection. 72 The observed cardiac microlesions were identical in appearance and size to those formed by $S$. 73 pneumoniae, were diffuse across the myocardium, and characterized by an absence of immune 74 cell infiltration and cell death by apoptosis and necroptosis. Furthermore, an enterococcal 75 determinant required for microlesion formation, DsbA, was identified. DsbA was additionally 76 shown to enhance cell death and promote suppression of the immune response during $E$. faecalis 77 infection of a cardiomyocyte cell line. 
Materials and Methods

C. elegans survival assay

E. faecalis strains were tested in C. elegans for survival as previously described [17]. The data is representative of 3 independent experiments. Nematode survival was plotted with the log-rank (Mantel-Cox) method using Prism 7 statistical software (GraphPad).

\section{Bacterial strains and growth analysis}

All E. faecalis strains used in this project are listed in Table S1. For growth analysis, overnight cultures were diluted to an $\mathrm{OD}_{600 \mathrm{~nm}}$ of 0.05 in brain heart infusion (BHI) medium (Difco) or serum $[18,19]$ in a volume of $200 \mu \mathrm{l} /$ well in a 96-well plate. The 96-well plate containing the diluted cultures were placed in a prewarmed $37^{\circ} \mathrm{C}$ BioTek Cytation 5 plate reader, and $\mathrm{OD}_{600 \mathrm{~nm}}$ measurements were taken every 10 minutes for 16 hours.

\section{Mouse peritonitis and cardiac microlesion model}

The mouse peritonitis model was performed as described previously [20]. The method for identifying and evaluating cardiac microlesion formation was conducted as described previously [11]. Briefly, female, $\sim 4-6$-week old $(\sim 25 g)$, ICR outbred mice were obtained from Envigo (Indianapolis, Indiana) and infected intraperitoneally with $3.8-4.2 \times 10^{8}$ CFUs of $E$. faecalis. Mouse hearts were harvested 24-48 hours post-infection at the point when the animals showed signs of severe sepsis indicated by ruffled fur, labored breathing and little movement. The hearts were formalin fixed and sent to The University of Texas Health Science Center Histopathology Laboratory for paraffin embedding, sectioning, and hematoxylin and eosin H\&E) staining. Paraffin embedded mouse hearts were first bisected and sectioned longitudinally. 20 sections were cut per block, and every $5^{\text {th }}$ section H\&E stained in order to measure the number and relative 
103 distribution of the microlesions. Cardiac microlesions were averaged across sections, and the

104 differences between mouse groups compared using Student's two-tailed t-test. Experiments were 105 performed in accordance with protocols approved by the Animal Welfare Committee of the 106 University of Texas Health Science Center at Houston AWC-19-0058.

107

108

109

110

112

114

115

\section{Invasion assay}

SVEC4-10 Endothelial Cells (ATCC) were seeded in 24 well plates at a cell density of $1.5 \times 10^{5}$ cells/ml. After 24 hours, the cells were washed three times with PBS and infected with E. faecalis at a MOI of 10 for 4 hours in antibiotic free Dulbecco's Modified Eagle's Medium (ATCC). The cells were then washed with Dulbecco's (1X) PBS three times, treated with trypsin, diluted in PBS, and plated for quantification. To measure intracellular numbers of $E$. faecalis, cells that were washed three times with Dulbecco's (1X) PBS 4 h.p.i., then treated with DMEM (ATCC) supplemented with $15 \mu \mathrm{g} / \mathrm{ml}$ vancomycin and $150 \mu \mathrm{g} / \mathrm{ml}$ gentamicin. 24 hours later, the wells were washed three times with Dulbecco's (1X) PBS, lysed using 0.1\% Triton-X-100, and diluted and plated for intracellular quantification. The ratio of invasion to attachment was used to calculate the percent of invasion. The experiments were conducted on 3 independent occasions and were performed using triplicate wells for each strain. Attachment and invasion values were averaged from each experiment, and statistically analyzed using Student's two-tailed t-test.

\section{Cytotoxicity and inflammatory cytokine panel microtiter plate reader assay}

HL-1 cardiomyocytes were obtained from Sigma-Aldrich and SVEC4-10 Endothelial Cells were obtained from ATCC. HL-1 cardiomyocytes (Sigma-Aldrich) were seeded into 24-well plates at a concentration of $5 \times 10^{5}$ cells $/ \mathrm{ml}$. 24 hours later, the cells were washed three times with Dulbecco's (1X) PBS, and infected with E. faecalis at a MOI of 10 for 4 hours in antibiotic free Claycomb medium. Supernatant was collected, and cytotoxicity assayed using CytoTox-ONE ${ }^{\text {TM }}$ 
128 Homogeneous Membrane Integrity Assay Kit (Promega). For HL-1 infected cardiomyocytes,

129 inflammatory chemokines and cytokines were additionally analyzed using a Mouse Cytokine

130 ELISA Plate Array III Colorimetric Assay (Signosis). The data represent an average of at least 3

131 independent experiments for each strain and were analyzed using Student's two-tailed t-test.

132

\section{Immunofluorescence microscopy}

134 Immunofluorescence microscopy was conducted as described previously [11]. Briefly, paraffin

embedded sections were treated with 1:100 rabbit Anti-mouse MLKL (phospho S345) antibody

[EPR9515(2)] in order to detect phosphorylated MLKL (Abcam), and 1:100 rat anti-mouse MLKL

antibody (Millipore-Sigma) for MLKL detection followed by treatment with 1:1000 chicken anti-

rabbit lgG $(\mathrm{H}+\mathrm{L})$ cross-adsorbed secondary Alexa Fluor 594 antibody (ThermoFisher), and 1:1000

goat anti-rat $\lg G(\mathrm{H}+\mathrm{L})$ secondary FITC antibody (ThermoFisher) respectively. A rabbit anti-

Enterococcus faecalis antibody (Abcam) was used at a concentration of 1:1000 to detect $E$.

141 faecalis followed by treatment with 1:1000 goat anti-rabbit IgG (H\&L), FITC conjugated antibody

142 (Invitrogen). A rat anti-PARP (MAB600) antibody (R\&DSystems) was used at a concentration of

$143 \quad 1: 100$ followed by treatment with 1:1000 goat anti-rat IgG $(\mathrm{H}+\mathrm{L})$ cross-adsorbed secondary Alexa

144 Fluor 594 antibody (ThermoFisher) to detect PARP. All stained sections were counterstained with

145 1:1000 DAPI (ThermoFisher) and mounted with Hardset $^{\mathrm{TM}}$ Antifade Mounting Medium.

146 Visualization of fluorescence was obtained using standard excitation and emission wavelengths

147 for Alexa 594, FITC, and DAPI on an Olympus FV3000RS high-speed, high-sensitivity, inverted 148 laser scanning confocal microscope.

\section{Generation of rabbit anti-DsbA antisera}

151 His-tagged recombinant DsbA was overexpressed in Rosetta-2(DE3) E. coli (Sigma-Aldrich) and 152 isolated by affinity purification via HisPur Nickel-NTA Resin (ThermoFisher) packed columns 
153 followed by Fast Protein Liquid Chromatography (FPLC) to obtain highly pure samples of

154 recombinant DsbA. Cocalico Biologicals (Reamstown, PA) generated anti-DsbA antisera by 155 immunizing rabbits with the recombinant protein.

\section{E. faecalis whole cell lysate and Western blot}

158 A $5 \mathrm{ml}$ culture of $E$. faecalis grown in $\mathrm{BHI}$ medium at $37^{\circ} \mathrm{C}$ for $15-16$ hours followed by a $1: 10$ 159 dilution in $5 \mathrm{ml}$ of fresh $\mathrm{BHI}$. After 3.5 hours to achieve exponential growth, the culture was 160 centrifuged at $6,010 \times \mathrm{G}$ and the supernatant removed. The pellet was resuspended in $1 \mathrm{ml}$ of 161 buffer $(10 \mathrm{mM}$ Tris/ $\mathrm{HCl} \mathrm{pH} 7.5$ and $200 \mathrm{mM} \mathrm{NaCl})$ containing cOmplete ${ }^{\mathrm{TM}}$, EDTA-free Protease 162 Inhibitor Cocktail (Roche). Following sonication of the pellet on ice, the sample was centrifuged 163 at $10,000 \times \mathrm{G}$ for 5 minutes and supernatant collected for electrophoresis. Total protein in the 164 whole cell lysate was measured using the DC Protein Assay (BioRad). Following the loading of $16510 \mu \mathrm{g}$ of cell lysate, electrophoresis and transfer of protein to a PVDF membrane, the membrane 166 was incubated overnight at $4^{\circ} \mathrm{C}$ in a $5 \%$ milk solution containing a 1:1000 dilution of rabbit anti167 DsbA antisera followed by incubation with a Goat anti-rabbit IgG HRP (ThermoFisher) secondary 168 antibody at a dilution of 1:10,000 at room temperature for 30 minutes prior to development with a 169 chemiluminescent kit (ThermoFisher). 
Results

172 Cardiac microlesions form during severe bacteremic $E$. faecalis infection. In previous work,

173 we implicated a previously uncharacterized E. faecalis thioredoxin known as DsbA (disulfide bond

174 forming protein $\mathrm{A}$ ) as a requirement for the stability of EntV, a secreted bacteriocin that inhibits

175 hyphal morphogenesis of the human fungal pathogen Candida albicans [21, 22]. To test if these

176 proteins had any effects on the fitness and pathogenicity of $E$. faecalis, we infected

177 Caenorhabditis elegans with $\Delta d s b A$ and $\Delta$ entV $E$. faecalis mutants and measured survival. As

178 shown in Figure $1 \mathrm{~A}$, loss of $d s b A$ significantly attenuated mortality in infected nematodes. Note

179 that the $\Delta d s b A$ mutant did not manifest different growth kinetics when grown in culture medium

180 compared to wild-type, ruling out a general growth defect (Figure S1A). Infection with the

181 complement, $\Delta d s b A:: d s b A$, partially rescued the $\Delta d s b A$ phenotype. The partial complementation

182 was attributed to the DsbA protein being produced at lower levels in the complemented strain

183 (Figure 1B and S2). No significant difference in nematode survival was observed following

184 infection with either the $\Delta$ entV mutant or its complement (Figure $1 \mathrm{~A}$ ). Considering that EntV is the

185 only known substrate of $E$. faecalis DsbA, the results suggest that DsbA has additional targets 186 that affect pathogenicity.

188 Based on these results, we wanted to know if loss of DsbA also affected virulence in a vertebrate animal model of $E$. faecalis infection. Therefore, the mutants were tested in a mouse model in

190 which animals were injected intra-peritoneally with $E$. faecalis resulting in generalized bacteremia

191 leading to mortality. Significant survival differences between the mice infected with the different

192 strains were subtle, but significant attenuation by the $d s b A$ mutant was observed when data from

193 three separate experiments were combined to generate an $\mathrm{n}$ close to 20 for each group (Figure

194 S3). To further study the contribution of $d s b A$, various organs were collected in order to determine 
195 if $E$. faecalis infection induced any strain-specific, pathological effects. To our surprise, cardiac

196 microlesions were discovered within the myocardium that were devoid of immune cell infiltrate 197 regardless of which $E$. faecalis strain was used (Figures 2A, B and S4). The microlesions

198 appeared to form within roughly the same time frame ( 24 h.p.i.) as cardiac microlesions that 199 have previously been reported to occur during invasive infection with S. pneumoniae [11].

201 To verify that the observed microlesions were the result of infection by $E$. faecalis, 202 immunofluorescent staining on paraffin embedded cardiac tissue sections from mice infected with

E. faecalis with a polyclonal anti-enterococcal IgG antibody was performed and revealed the presence of dense aggregates of $E$. faecalis within the microlesions (Figure 2C). The presence of bacterial DNA was further verified by counterstaining with DAPI, revealing an accumulation of non-cardiomyocyte nuclear DNA within microlesions (Figure 2D). To determine the contribution of DsbA, cardiac microlesions found on hematoxylin and eosin stained paraffin embedded cardiac sections were quantified following infection with $\Delta d s b A$. A significant attenuation in microlesion formation during infection with $\Delta d s b A$ was observed relative to wild-type, while no significant difference was observed in mice infected with the $d s b A$ complement (Figure 2E). Furthermore, when the sizes of the cardiac microlesions were compared, the wild-type lesions were significantly 212 larger than those found in the $\Delta d s b A$ infected mice (Figure 2F). Importantly, the size of the microlesions appeared to correlate with level of $d s b A$ expression observed in Western blots of $E$.

214 faecalis whole cell lysates (Figure $1 \mathrm{~B}$ ), with the $\triangle d s b A:: d s b A$ strain manifesting an in-between 215 phenotype to that of the $\Delta d s b A$ and wild-type strains, consistent with partial complementation. It 216 is possible that less microlesion formation occurred with the $\Delta d s b A$ strain due poor growth in the 217 blood, but we do not favor this model as in vitro growth curves in serum showed no defect (Figure 218 S1B). In conclusion, these data demonstrate that cardiac microlesion formation occurs during 219 systematic infection with E. faecalis and is dependent on DsbA. 
221 Apoptosis and necroptosis contribute to cardiomyocyte cell death within microlesions. In 222 previous studies of pneumococcal cardiac microlesion formation, apoptosis was found to be 223 associated with microlesion formation in mice [11]. A subsequent study determined that 224 cardiomyocyte cell death occurs following exposure to pneumolysin (ply) and pneumococcal 225 pyruvate oxidase $(s p x B)$ derived hydrogen peroxide [14]. Based on these observations and the fact that $E$. faecalis lacks ply and $s p x B$ homologs, we sought to determine whether $E$. faecalis could promote cell death of cardiomyocytes and identify the mechanism. To determine if apoptosis contributes to $E$. faecalis microlesion formation, we performed immunofluorescent microscopy on heart sections of infected mice to detect PARP [Poly(ADP-ribose) Polymerase], an enzyme that is proteolytically cleaved by the effector Caspase-3, and is one hallmark event of apoptosis. In agreement with previous reports, markers of apoptosis were observed to be concentrated at sites of microlesion formation (Figure 3A and S5).

In addition to apoptosis, we hypothesized that necroptosis also contributes to $E$. faecalis mediated cardiac microlesion formation. Necroptosis is a programmed form of necrotic cell death that is generally inflammatory, and has been shown to contribute to the formation of $S$. pneumoniae microlesions within the myocardium of acutely ill mice and non-human primates [12]. Importantly, necroptosis is considered a key cell death pathway in cardiomyocytes during ischemiareperfusion injury and acute coronary syndrome [23-25]. To test the hypothesis that necroptosis contributes to the formation of E. faecalis microlesions, we stained for markers of necroptosis [26], such as mixed lineage kinase domain-like pseudokinase (MLKL) and phosphorylated MLKL, on cardiac tissue sections obtained from infected mice. As a result, we detected concentrated signals within the microlesions (Fig 3B and Figure S6) that suggest that necroptosis does indeed contribute to $E$. faecalis cardiac microlesion formation. 
246 To further look at the effects of $E$. faecalis and the $d s b A$ mutant on heart cells, we infected HL-1 247 cardiomyocytes and subsequently measured lactate dehydrogenase (LDH) release into the cell 248 culture supernatant to assess cell death. As shown in Figure 3C, cell death of the cardiomyocytes 249 is significantly decreased following exposure to the dsbA mutant compared to wild-type 250 suggesting that DsbA contributes to cardiac cell death (Figure $3 C$ ). While E. faecalis promoted death of the cardiomyocytes, antibiotic protection assays indicated that both the wild-type and 252 $d s b A$ mutant bacteria were unable to invade cardiomyocytes relative to endothelial cells (Figure 3D). Taken together, these results suggest that during bacteremia, E. faecalis is able to translocate across the vascular endothelium in order to enter the myocardial tissue. Once there, E. faecalis is unable to invade cardiomyocytes, but can form microcolonies that promote necroptosis in addition to apoptosis. Whether other cell death pathways additionally contribute will be fodder for future investigations.

258 Cardiomyocyte inflammatory cytokine response is suppressed following exposure to $E$.

faecalis. Cardiac microlesions are characterized by bacteria filled vacuoles that are largely devoid 260 of immune cell infiltrate $[11,27]$. Previous efforts to explain the lack of an immune response to 261 pneumococcal microlesions have yielded interesting insights. For example, S. pneumoniae within microlesions are thought to subvert the host response through a biofilm-mediated mechanism 263 involving the pneumolysin mediated killing of resident macrophages, thus creating a more 264 permissive environment for pneumococcal growth and survival [13]. There are also examples of 265 E. faecalis subverting the immune response. In previous work, it was shown that $E$. faecalis can survive within mouse peritoneal macrophages for extended periods of time [28] and can also resist 267 phagosome acidification and autophagy [29]. Additionally, E. faecalis was shown to activate 268 Phosphatidylinositol 3-Kinase signaling that inhibits apoptosis [30]. Because E. faecalis does not 
270 immune cells, we wanted to examine the immune response to $E$. faecalis within cardiomyocytes.

271 Therefore, following infection with E. faecalis, inflammatory chemokine and cytokine levels were 272 measured. Wild-type E. faecalis was discovered to promote an immune quiescent response, while 273 the $d s b A$ mutant caused a more inflammatory immune response (Figure 4A and Table S2). 274 Specifically, infection of cardiomyocytes with wild-type increased the expression of two pro275 inflammatory cytokines IL-4 and b-NGF cytokines [31, 32]. Modest increases were also observed 276 in VEGF, an important determinant of sepsis morbidity and mortality [33], and GM-CSF that 277 serves as an immunomodulatory cytokine [34]. In contrast, cardiomyocytes infected with $\Delta d s b A$ 278 led to increases in Rantes, IFNr, MIP-1a, and IL-12, with modest increases also observed in Leptin 279 and G-CSF. With the exception of G-CSF, an immunomodulatory cytokine with roles in cardiac 280 cell generation and repair [35, 36], all of the other aforementioned cytokines are pro-inflammatory 281 [37-40]. These results implicate the contribution of specific host signaling pathways that are 282 normally suppressed in the presence of DsbA during infection, as modeled in Figure 4B. 283 Collectively, these data suggest that $E$. faecalis is capable of suppressing the host cardiomyocyte 284 immune response. 
Discussion

287 In this work, we demonstrated for the first time that cardiac microlesions form during severe $E$. 288 faecalis bacteremic infection of mice. Importantly, microlesions occurred despite E. faecalis not 289 encoding homologs of the pneumococcal virulence determinants implicated in microlesion 290 formation. These data suggest that $E$. faecalis may be expressing functionally redundant proteins 291 that serve to promote similar interactions to S. pneumoniae in mediating microlesion formation, 292 such as CbpA and pneumolysin, which facilitate vascular endothelial cell translocation and 293

cardiomyocyte cell death, respectively. Indeed, while $E$. faecalis does not encode a CbpA homolog, it does encode other proteins capable of binding host extracellular matrix proteins.

These include EfbA, which binds fibronectin [41], ACE, which binds laminin receptor [42], and Ebp 296 pili that bind fibrinogen [43].

Interestingly, we did discover one factor, DsbA, that contributes to microlesion formation and inhibits the cardiomyocyte inflammatory immune response, as modeled in Figure 4B. Dsb proteins work to catalyze disulfide bond formation in proteins and have been best studied in Gram-negative bacteria $[44,45]$. While Dsb proteins have established importance in the biology of certain Grampositive bacteria, Dsb proteins are not well studied in Firmicutes like E. faecalis and have generally not been considered important [44]. Most of these species secrete few proteins containing cysteines and some, notably S. pneumoniae, do not encode any thiol-disulfide oxidoreductases $[46,47]$. Thus, it will be of interest to further investigate the role of Dsb proteins in these species.

307 Our prior work established EntV as a DsbA substrate, currently the only one identified [21]. 308 However, loss of EntV did not reduce pathogenicity in the worm model (Figure 1A), and exposure 309 to EntV, even at very high concentrations, was non-toxic to mammalian cells [22]. As such, we do 
310 not favor EntV as being the source of DsbA's effects. While the specific E. faecalis proteins

311 involved in modulating the host immune response are unknown, we speculate that the

312 Fibronectin-binding protein $(\mathrm{Efb})$ and Choline binding protein may contribute to cardiac

313 microlesion formation by serving as functionally redundant proteins to pneumococcal CbpA, a

314 virulence determinant known to be integral to microlesion formation [11]. Moreover, both proteins

315 contain 5 cysteines and are expressed extracellularly. Thus, our future research efforts will be 316 geared toward identifying additional substrates of DsbA and to determining their contribution to 317 microlesion formation.

319 Pneumococcal ChoP interactions with host PAFr were determined to be a prerequisite for 320 vascular endothelial cell invasion during IPD. In light of our observations of $E$. faecalis invasion of 321 vascular endothelial cells despite an absence of this wall teichoic acid structure, it is possible that 322 another host receptor is required for endothelial cell invasion. Interestingly, recent studies focused on bacterial cell wall entry and signaling in eukaryotic cells have identified a PAFr-independent 324 mechanism of tissue invasion that is actin-dependent and engenders Rac1, Cdc42, and phosphatidylinositol 3-kinase $(\mathrm{PI} K \mathrm{~K})$ signaling leading to cell wall internalization via a macropinoctytosis-like pathway [48]. Whether such a pathway contributes to microlesion formation is unknown. In the future, it will be of interest to study the mechanisms of tissue invasion and the host signaling pathways involved.

Although the role of cardiac microlesion formation in humans during invasive disease remains unclear, cardiac microlesion formation is becoming increasingly appreciated as a risk factor [49].

332 To our knowledge, no one has investigated whether cardiac microlesions form in humans during 333 enterococcal infection. We speculate that formation occurs and contributes to cardiac 334 abnormalities or death in patients with enterococcal infective endocarditis or sepsis [1, 3-9]. This 
335 study builds on our understanding of cardiac microlesions by establishing $E$. faecalis as another 336 etiological agent capable of causing them in a vertebrate animal model. Considering that $E$. 337 faecalis microlesion pathology appears identical to that caused by S. pneumoniae, it is likely that 338 scar formation occurs following resolution of infection, potentially predisposing individuals to future 339 adverse cardiac events [11]. Taken together, these data highlight the need for further investigation 340 in order to understand the impact of microlesion formation on the heart and mortality during 341 invasive $E$. faecalis infection. 
342

343

344

345

346

347

348

349

\section{Enterococcus faecalis Strains}

\begin{tabular}{|l|l|l|l|l|}
\hline Strain & Genotype & Characteristics & Parent & Source \\
\hline OG1RF & Wild-type & $\mathrm{Gel}^{+} \mathrm{Spr}^{+} \mathrm{Rf}^{\mathrm{R}} \mathrm{Fa}^{\mathrm{R}}$ & $\mathrm{OG} 1$ & {$[50]$} \\
\hline AOBD1 & $\Delta d s b A$ & $\mathrm{Gel}^{+} \mathrm{Spr}^{+} \mathrm{Rf}^{\mathrm{R}} \mathrm{Fa}^{\mathrm{R}}$ & $\mathrm{OG} 1$ & {$[21]$} \\
\hline AOBD2 & $\Delta d s b A:: d s b A$ & $\mathrm{Gel}^{+} \mathrm{Spr}^{+} \mathrm{Rf}^{\mathrm{R}} \mathrm{Fa}^{\mathrm{R}}$ & $\mathrm{OG} 1$ & {$[21]$} \\
\hline
\end{tabular}

Table S2. Cytokine Profiling Expression Data 
bioRxiv preprint doi: https://doi.org/10.1101/2020.01.14.906669; this version posted June 5, 2020. The copyright holder for this preprint (which was not certified by peer review) is the author/funder. All rights reserved. No reuse allowed without permission.

Cytokine Profiling - Fold Change Data for 3 independent Experiments

4 hour infection with an $\mathrm{MOI}=10$

\begin{tabular}{|l|c|c|c|c|c|}
\hline & Exp. 1 & Exp. 2 & Exp. 3 & & \\
\hline & OG1RF & OG1RF & OG1RF & Average & Std. Dev \\
\hline TNF-a & $-7 \%$ & $7 \%$ & $-2 \%$ & $-1 \%$ & $7 \%$ \\
\hline IGF-1 & $2 \%$ & $-7 \%$ & $4 \%$ & $0 \%$ & $6 \%$ \\
\hline VEGF & $23 \%$ & $4 \%$ & $-2 \%$ & $8 \%$ & $13 \%$ \\
\hline IL-6 & $7 \%$ & $5 \%$ & $1 \%$ & $4 \%$ & $3 \%$ \\
\hline FGFb & $11 \%$ & $-28 \%$ & $0 \%$ & $-5 \%$ & $20 \%$ \\
\hline IFNr & $5 \%$ & $3 \%$ & $-11 \%$ & $-1 \%$ & $9 \%$ \\
\hline EGF & $-10 \%$ & $33 \%$ & $2 \%$ & $8 \%$ & $23 \%$ \\
\hline Leptin & $0 \%$ & $-16 \%$ & $-1 \%$ & $-6 \%$ & $9 \%$ \\
\hline IL-1a & $-7 \%$ & $-14 \%$ & $4 \%$ & $-6 \%$ & $9 \%$ \\
\hline IL-1b & $-8 \%$ & $-22 \%$ & $18 \%$ & $-4 \%$ & $20 \%$ \\
\hline G-CSF & $1 \%$ & $-22 \%$ & $2 \%$ & $-6 \%$ & $14 \%$ \\
\hline GM-CSF & $-10 \%$ & $19 \%$ & $14 \%$ & $7 \%$ & $16 \%$ \\
\hline MCP-1 & $-7 \%$ & $-8 \%$ & $11 \%$ & $-1 \%$ & $11 \%$ \\
\hline M1P-1a & $3 \%$ & $3 \%$ & $-17 \%$ & $-4 \%$ & $12 \%$ \\
\hline SCF & $-15 \%$ & $5 \%$ & $-3 \%$ & $-5 \%$ & $10 \%$ \\
\hline Rantes & $-13 \%$ & $19 \%$ & $-7 \%$ & $0 \%$ & $17 \%$ \\
\hline PDGF-BB & $-16 \%$ & $8 \%$ & $7 \%$ & $0 \%$ & $13 \%$ \\
\hline b-NGF & $-20 \%$ & $12 \%$ & $17 \%$ & $3 \%$ & $20 \%$ \\
\hline IL-17a & $-22 \%$ & $-4 \%$ & $9 \%$ & $-5 \%$ & $16 \%$ \\
\hline IL-2 & $-9 \%$ & $-10 \%$ & $-5 \%$ & $-8 \%$ & $3 \%$ \\
\hline IL-4 & $12 \%$ & $-1 \%$ & $9 \%$ & $7 \%$ & $7 \%$ \\
\hline IL-10 & $11 \%$ & $-13 \%$ & $-3 \%$ & $-2 \%$ & $12 \%$ \\
\hline Resistin & $-13 \%$ & $-3 \%$ & $-6 \%$ & $-8 \%$ & $5 \%$ \\
\hline IL-12 & $-3 \%$ & $0 \%$ & $-17 \%$ & $-7 \%$ & $9 \%$ \\
\hline CCL11 & $-9 \%$ & $-25 \%$ & $3 \%$ & $-10 \%$ & $14 \%$ \\
\hline CCL21 & $-17 \%$ & $0 \%$ & $-5 \%$ & $-7 \%$ & $9 \%$ \\
\hline IL-3 & $8 \%$ & $3 \%$ & $5 \%$ & $5 \%$ & $2 \%$ \\
\hline IL-13 & $-7 \%$ & $-7 \%$ & $10 \%$ & $-1 \%$ & $10 \%$ \\
\hline IL-21 & $-24 \%$ & $-14 \%$ & $1 \%$ & $-12 \%$ & $13 \%$ \\
\hline IL-22 & $-18 \%$ & $-19 \%$ & $-12 \%$ & $-16 \%$ & $4 \%$ \\
\hline CXCL10 & $-12 \%$ & $-10 \%$ & $2 \%$ & $-7 \%$ & $8 \%$ \\
\hline CXCL1 & $-5 \%$ & $-4 \%$ & $-13 \%$ & $-7 \%$ & $5 \%$ \\
\hline
\end{tabular}

*OG1RF ordered from Largest to smallest

\begin{tabular}{l|c|c} 
& OG1RF & $\boldsymbol{\Delta} \mathbf{d s} \boldsymbol{d} \boldsymbol{A}$ \\
\hline EGF & $8 \%$ & $3 \%$ \\
\hline VEGF & $8 \%$ & $1 \%$ \\
\hline GM-CSF & $7 \%$ & $1 \%$ \\
\hline IL-4 & $7 \%$ & $-12 \%$ \\
\hline IL-3 & $5 \%$ & $-1 \%$ \\
\hline IL-6 & $4 \%$ & $2 \%$ \\
\hline b-NGF & $3 \%$ & $-8 \%$ \\
\hline PDGF-BB & $0 \%$ & $-2 \%$ \\
\hline IGF-1 & $0 \%$ & $-6 \%$ \\
\hline Rantes & $0 \%$ & $4 \%$ \\
\hline TNF-a & $-1 \%$ & $-2 \%$ \\
\hline IFNr & $-1 \%$ & $4 \%$ \\
\hline IL-13 & $-1 \%$ & $-3 \%$ \\
\hline MCP-1 & $-1 \%$ & $-2 \%$ \\
\hline IL-10 & $-2 \%$ & $-2 \%$ \\
\hline M1P-1a & $-4 \%$ & $3 \%$ \\
\hline IL-1b & $-4 \%$ & $-13 \%$ \\
\hline SCF & $-5 \%$ & $-7 \%$ \\
\hline FGFb & $-5 \%$ & $-8 \%$ \\
\hline IL-17a & $-5 \%$ & $-14 \%$ \\
\hline IL-1a & $-6 \%$ & $-7 \%$ \\
\hline Leptin & $-6 \%$ & $0 \%$ \\
\hline G-CSF & $-6 \%$ & $2 \%$ \\
\hline CXCL10 & $-7 \%$ & $-11 \%$ \\
\hline IL-12 & $-7 \%$ & $1 \%$ \\
\hline CXCL1 & $-7 \%$ & $-3 \%$ \\
\hline CCL21 & $-7 \%$ & $-9 \%$ \\
\hline Resistin & $-8 \%$ & $-10 \%$ \\
\hline IL-2 & $-8 \%$ & $-11 \%$ \\
\hline CCL11 & $-10 \%$ & $-12 \%$ \\
\hline IL-21 & $-12 \%$ & $-9 \%$ \\
\hline IL-22 & $-16 \%$ & $-5 \%$ \\
\hline & & \\
\hline
\end{tabular}

\begin{tabular}{|l|c|c|c|c|c|}
\hline & Exp. 1 & Exp. 2 & Exp. 3 & & \\
\hline & $\boldsymbol{\Delta d} \mathbf{d} \boldsymbol{A}$ & $\boldsymbol{\Delta} \mathbf{d s} \boldsymbol{B} \boldsymbol{A}$ & $\boldsymbol{\Delta d s} \mathbf{A}$ & Average & Std. Dev \\
\hline TNF-a & $-2 \%$ & $5 \%$ & $-8 \%$ & $-2 \%$ & $6 \%$ \\
\hline IGF-1 & $-15 \%$ & $-7 \%$ & $3 \%$ & $-6 \%$ & $9 \%$ \\
\hline VEGF & $2 \%$ & $1 \%$ & $0 \%$ & $1 \%$ & $1 \%$ \\
\hline IL-6 & $0 \%$ & $13 \%$ & $-8 \%$ & $2 \%$ & $10 \%$ \\
\hline FGFb & $-7 \%$ & $-15 \%$ & $-2 \%$ & $-8 \%$ & $7 \%$ \\
\hline IFNr & $16 \%$ & $7 \%$ & $-12 \%$ & $4 \%$ & $14 \%$ \\
\hline EGF & $-8 \%$ & $31 \%$ & $-12 \%$ & $3 \%$ & $24 \%$ \\
\hline Leptin & $13 \%$ & $-8 \%$ & $-4 \%$ & $0 \%$ & $11 \%$ \\
\hline IL-1a & $-17 \%$ & $-10 \%$ & $7 \%$ & $-7 \%$ & $12 \%$ \\
\hline IL-1b & $-20 \%$ & $-19 \%$ & $-1 \%$ & $-13 \%$ & $11 \%$ \\
\hline G-CSF & $-12 \%$ & $14 \%$ & $3 \%$ & $2 \%$ & $13 \%$ \\
\hline GM-CSF & $-18 \%$ & $18 \%$ & $2 \%$ & $1 \%$ & $18 \%$ \\
\hline MCP-1 & $-20 \%$ & $-9 \%$ & $23 \%$ & $-2 \%$ & $22 \%$ \\
\hline M1P-1a & $-6 \%$ & $3 \%$ & $13 \%$ & $3 \%$ & $10 \%$ \\
\hline SCF & $-10 \%$ & $-20 \%$ & $10 \%$ & $-7 \%$ & $15 \%$ \\
\hline Rantes & $-3 \%$ & $20 \%$ & $-4 \%$ & $4 \%$ & $14 \%$ \\
\hline PDGF-BB & $-23 \%$ & $15 \%$ & $4 \%$ & $-2 \%$ & $20 \%$ \\
\hline b-NGF & $-16 \%$ & $-10 \%$ & $1 \%$ & $-8 \%$ & $9 \%$ \\
\hline IL-17a & $-45 \%$ & $-1 \%$ & $4 \%$ & $-14 \%$ & $27 \%$ \\
\hline IL-2 & $-35 \%$ & $-1 \%$ & $1 \%$ & $-11 \%$ & $20 \%$ \\
\hline IL-4 & $-17 \%$ & $-21 \%$ & $2 \%$ & $-12 \%$ & $13 \%$ \\
\hline IL-10 & $0 \%$ & $-2 \%$ & $-4 \%$ & $-2 \%$ & $2 \%$ \\
\hline Resistin & $-19 \%$ & $-6 \%$ & $-6 \%$ & $-10 \%$ & $7 \%$ \\
\hline IL-12 & $-13 \%$ & $14 \%$ & $1 \%$ & $1 \%$ & $14 \%$ \\
\hline CCL11 & $-13 \%$ & $-30 \%$ & $7 \%$ & $-12 \%$ & $18 \%$ \\
\hline CCL21 & $-33 \%$ & $4 \%$ & $3 \%$ & $-9 \%$ & $21 \%$ \\
\hline IL-3 & $-8 \%$ & $2 \%$ & $3 \%$ & $-1 \%$ & $6 \%$ \\
\hline IL-13 & $-25 \%$ & $-3 \%$ & $19 \%$ & $-3 \%$ & $22 \%$ \\
\hline IL-21 & $-23 \%$ & $-10 \%$ & $6 \%$ & $-9 \%$ & $15 \%$ \\
\hline IL-22 & $-26 \%$ & $-7 \%$ & $18 \%$ & $-5 \%$ & $22 \%$ \\
\hline CXCL10 & $-14 \%$ & $-17 \%$ & $-2 \%$ & $-11 \%$ & $8 \%$ \\
\hline CXCL1 & $-12 \%$ & $-15 \%$ & $17 \%$ & $-3 \%$ & $17 \%$ \\
\hline
\end{tabular}




\section{Figure Legends}

Figure 1. $d s b A$ deficient $E$. faecalis show reduced virulence in a $C$. elegans survival assay. (A) L4 stage C. elegans were infected with E. faecalis. This figure is representative of 3 independent experiments with 30 worms/group. ${ }^{* * * *}$ Asterisks indicate a $P<0.0001,{ }^{* *}$ a $P<0.01$ when compared to OG1RF following pairwise analysis by log-rank (Mantel-Cox). (B) Western blot performed by SDS-PAGE separation of $E$. faecalis whole cell lysates followed by hybridization of DsbA using rabbit anti-DsbA antisera. Equal loading is ensured by visualization of a non-specific band at $40 \mathrm{kDa}$. Image is representative of 3 independent Western blot experiments.

\section{Figure 2. Cardiac microlesions form during invasive $E$. faecalis infection and are} attenuated by loss of $d s b A$. Cardiac microlesions were observed using light microscopy of hematoxylin and eosin (H\&E) stained paraffin embedded cardiac sections at 40X magnification (A), and 100X magnification (B). Immunofluorescent detection of wild-type E. faecalis was imaged using a 20X objective at $3 \mathrm{X}$ zoom (total of $60 \mathrm{X}$ magnification), a FITC conjugated antienterococcal antibody (C), and DAPI for DNA staining (D). Total microlesion counts (E), and size measurements $(F)$, were obtained by quantification using light microscopy of H\&E stained paraffin embedded cardiac sections from mice infected for 24-48 hours with $E$. faecalis (OG1RF $n=14$, $\triangle d s b A \mathrm{n}=14, \Delta d s b A:: d s b A \mathrm{n}=14)$. The data were averaged from 3 independent experiments and analyzed using Student's two-tailed t-test ( ${ }^{*}$ indicate a $P<0.05,{ }^{* *} P<0.01,{ }^{* * *}$ a $P<0.001$ ).

Figure 3. Necroptosis contributes to cardiotoxicity within cardiac microlesions. (A) Immunofluorescent microscopy of paraffin embedded cardiac cross sections at $20 \mathrm{X}$ were performed to detect E. faecalis using FITC conjugated anti-faecalis antibodies, and apoptosis using Alexa 594 conjugated anti-PARP antibodies at sites of microlesion formation, while DAPI 
was used to stain nuclei. (B) Immunofluorescent microscopy of paraffin embedded cardiac cross sections at 20X were performed to detect MLKL using FITC conjugated anti-MLKL antibodies, and

Alexa 594 conjugated anti-phosphorylated MLKL antibodies at sites of microlesion formation,

while DAPI was used to stain nuclei. (C) E. faecalis induced cell death in HL-1 cardiomyocytes 4

381 hours post-infection (h.p.i.) as determined by measuring lactate dehydrogenase. The data 382 represent an average of 3 independent experiments and were analyzed using Student's two-tailed t-test ( ${ }^{*}$ indicate a $P<0.05$ ). (D) Comparison of OG1RF and $\Delta d s b A$ invasion rates into $\mathrm{HL}-1$ cardiomyocytes and SVEC endothelial cells. The graph represents data from 3 independent experiments that were analyzed using Student's two-tailed t-test.

Figure 4. Wild-type E. faecalis suppresses the cardiomyocyte inflammatory response in-

vitro. (A) HL-1 cardiomyocytes exposed to $E$. faecalis promote a suppressed inflammatory response while $\Delta d s b A$ infected cardiomyocytes yield a more inflammatory response. Data was obtained from the fold-expression change analysis (relative to uninfected controls) of at least 3 independent experiments for each strain. (B) Model of $E$. faecalis mediated suppression of the host cardiomyocyte immune response and effect on microlesion size. Wild-type E. faecalis is postulated to downregulate the cardiomyocyte immune response more effectively than the $d s b A$ mutant resulting in more cell death and increased lesion size. However, both strains cause cell death by apoptosis and necroptosis and inhibit immune cell infiltration.

Supplemental Figure 1. E. faecalis growth curve. (A) E. faecalis strains were grown for 15 hours in $\mathrm{BHI}$ and absorbance $\left(\mathrm{OD}_{600 \mathrm{~nm}}\right)$ measured every 10 minutes for 16 hours. (B) E. faecalis grown in serum and absorbance $\left(\mathrm{OD}_{600 \mathrm{~nm}}\right)$ measured every 10 minutes for 16 hours. Figures are representative of 3 independent experiments. 
Supplemental Figure 2. Coomassie stained SDS-PAGE gel of E. faecalis whole cell lysates

separated by electrophoresis. (A) SDS-PAGE separation of E. faecalis whole cell lysates was

stained with Coomassie to verify that each well received $10 \mu \mathrm{g} /$ lane of whole cell lysate.

Coomassie stained gel is the loading control for the Western blot in Figure 1B.

Supplemental Figure 3. DsbA is required for E. faecalis pathogenesis. (A) Mice $(n=17-20)$

challenged with $3.8-4.2 \times 10^{8} \mathrm{CFUs}$ of $E$. faecalis in a mouse model of peritonitis were assessed 409

for survival over 96 hours. Mice infected with $\triangle d s b A$ E. faecalis lived significantly longer $(P=$ 410

0.0051) than mice challenged with wild-type or the complemented strain (no significant difference). Survival curve was calculated from 3 independent experiments using log-rank 412 analysis.

Supplemental Figure 4. (A) 40X magnification image of cardiac microlesion formation in mice infected with $\triangle d s b A$. (B) 100X image of cardiac microlesion in Figure S4A. (C) 40X magnification image of cardiac microlesion formation in mice infected with $\Delta d s b A:: d s b A$. (D) $100 \mathrm{X}$ image of cardiac microlesion in Figure S4C.

\section{Supplemental Figure 5. Necroptosis contributes to cardiotoxicity within cardiac}

microlesions. (A) Immunofluorescent microscopy of paraffin embedded cardiac cross sections using a 20X objective at $4 \mathrm{X}$ Zoom (80X total magnification) were performed to detect MLKL using FITC conjugated anti-MLKL antibodies, and Alexa 594 conjugated anti-phosphorylated MLKL antibodies at sites of microlesion formation, while DAPI was used to stain nuclei.

Supplemental Figure 6. Apoptosis contributes to cell death within cardiac microlesions. 
427 objective at $4 \mathrm{X}$ Zoom (80X total magnification) were performed to detect E. faecalis using FITC 428 conjugated anti-E. faecalis antibodies, and Alexa 594 conjugated anti-PARP antibodies at sites of 429 microlesion formation, while DAPI was used to stain nuclei.

References

434 1. Chirouze $C$, Athan E, Alla F, et al. Enterococcal endocarditis in the beginning of the 21st century: analysis from the International Collaboration on Endocarditis-Prospective Cohort Study. Clin Microbiol Infect 2013; 19:1140-7.

2. Agudelo Higuita NI, Huycke MM. Enterococcal Disease, Epidemiology, and Implications for 438 Treatment. In: Gilmore MS, Clewell DB, Ike Y, Shankar N, eds. Enterococci: From Commensals to Leading Causes of Drug Resistant Infection. Boston, 2014.

3. Cabell CH, Jollis JG, Peterson GE, et al. Changing patient characteristics and the effect on mortality in endocarditis. Arch Intern Med 2002; 162:90-4.

4. Garvey GJ, Neu HC. Infective endocarditis--an evolving disease. A review of endocarditis at 443 the Columbia-Presbyterian Medical Center, 1968-1973. Medicine (Baltimore) 1978; 57:105-27. endocarditis; clinical, pathologic and therapeutic consideration of 33 cases. Circulation 1954; 10:173-94.

447 6. Jawetz E, Sonne M. Penicillin-streptomycin treatment of enterococcal endocarditis. A re448 evaluation. N Engl J Med 1966; 274:710-5.

449 7. Lerner PI, Weinstein L. Infective endocarditis in the antibiotic era. N Engl J Med 1966; 274:38845093 concl. 
451 8. Olaison L, Schadewitz K, Swedish Society of Infectious Diseases Quality Assurance Study 452 Group for E. Enterococcal endocarditis in Sweden, 1995-1999: can shorter therapy with 453 aminoglycosides be used? Clin Infect Dis 2002; 34:159-66.

454 9. Pelletier LL, Jr., Petersdorf RG. Infective endocarditis: a review of 125 cases from the University 455 of Washington Hospitals, 1963-72. Medicine (Baltimore) 1977; 56:287-313.

456 10. Garsin DA, Frank KL, Silanpaa J, et al. Pathogenesis and Models of Enterococcal Infection. 457 In: Gilmore MS, Clewell DB, Ike Y, Shankar N, eds. Enterococci: From Commensals to Leading 458 Causes of Drug Resistant Infection. Boston, 2014.

459 11. Brown AO, Mann B, Gao G, et al. Streptococcus pneumoniae translocates into the 460 myocardium and forms unique microlesions that disrupt cardiac function. PLoS Pathog 2014; $461 \quad 10: e 1004383$.

462 12. Reyes LF, Restrepo MI, Hinojosa CA, et al. Severe Pneumococcal Pneumonia Causes Acute 463 Cardiac Toxicity and Subsequent Cardiac Remodeling. Am J Respir Crit Care Med 2017; 464 196:609-20.

465 13. Shenoy AT, Brissac T, Gilley RP, et al. Streptococcus pneumoniae in the heart subvert the 466 host response through biofilm-mediated resident macrophage killing. PLoS Pathog 2017; 467 13:e1006582.

468 14. Brissac T, Shenoy AT, Patterson LA, Orihuela CJ. Cell Invasion and Pyruvate Oxidase469 Derived H2O2 Are Critical for Streptococcus pneumoniae-Mediated Cardiomyocyte Killing. Infect $470 \quad$ Immun 2018; 86.

471 15. Makara MA, Hoang KV, Ganesan LP, et al. Cardiac Electrical and Structural Changes During 472 Bacterial Infection: An Instructive Model to Study Cardiac Dysfunction in Sepsis. J Am Heart 473 Assoc 2016; 5.

474 16. Headley CA, Gerberick A, Mehta S, et al. Nontuberculous mycobacterium M. avium infection 475 predisposes aged mice to cardiac abnormalities and inflammation. Aging Cell 2019; $18: e 12926$. 
476 17. Garsin DA, Sifri CD, Mylonakis E, et al. A simple model host for identifying Gram-positive 477 virulence factors. Proc Natl Acad Sci U S A 2001; 98:10892-7.

478 18. Nallapareddy SR, Murray BE. Role played by serum, a biological cue, in the adherence of 479 Enterococcus faecalis to extracellular matrix proteins, collagen, fibrinogen, and fibronectin. J $480 \quad$ Infect Dis 2008; 197:1728-36.

481 19. Teng F, Singh KV, Bourgogne A, Zeng J, Murray BE. Further characterization of the epa gene 482 cluster and Epa polysaccharides of Enterococcus faecalis. Infect Immun 2009; 77:3759-67.

483 20. Singh KV, Qin X, Weinstock GM, Murray BE. Generation and testing of mutants of 484 Enterococcus faecalis in a mouse peritonitis model. J Infect Dis 1998; 178:1416-20.

485 21. Armand O. Brown CEG, Melissa R. Cruz, Michael C. Lorenz, Danielle A. Garsin. Antifungal 486 activity of the Enterococcus faecalis peptide EntV requires protease cleavage and disulfide bond 487 formation. Mbio 2019; 10:1-13.

488 22. Graham CE, Cruz MR, Garsin DA, Lorenz MC. Enterococcus faecalis bacteriocin EntV inhibits 489 hyphal morphogenesis, biofilm formation, and virulence of Candida albicans. Proc Natl Acad Sci 490 U S A 2017; 114:4507-12.

491 23. Zhang T, Zhang Y, Cui M, et al. CaMKII is a RIP3 substrate mediating ischemia- and oxidative 492 stress-induced myocardial necroptosis. Nat Med 2016; 22:175-82.

493 24. Linkermann A, Hackl MJ, Kunzendorf U, Walczak H, Krautwald S, Jevnikar AM. Necroptosis 494 in immunity and ischemia-reperfusion injury. Am J Transplant 2013; 13:2797-804.

495 25. Linkermann A, Brasen JH, Darding M, et al. Two independent pathways of regulated necrosis 496 mediate ischemia-reperfusion injury. Proc Natl Acad Sci U S A 2013; 110:12024-9.

497 26. Kitur K, Wachtel S, Brown A, et al. Necroptosis Promotes Staphylococcus aureus Clearance 498 by Inhibiting Excessive Inflammatory Signaling. Cell Rep 2016; 16:2219-30. 
499 27. Gilley RP, Gonzalez-Juarbe N, Shenoy AT, et al. Infiltrated Macrophages Die of Pneumolysin500 Mediated Necroptosis following Pneumococcal Myocardial Invasion. Infect Immun 2016; 84:145750169.

502 28. Gentry-Weeks CR, Karkhoff-Schweizer R, Pikis A, Estay M, Keith JM. Survival of 503 Enterococcus faecalis in mouse peritoneal macrophages. Infect Immun 1999; 67:2160-5.

504 29. Zou J, Shankar N. The opportunistic pathogen Enterococcus faecalis resists phagosome 505 acidification and autophagy to promote intracellular survival in macrophages. Cell Microbiol 2016; $506 \quad$ 18:831-43.

507 30. Zou J, Shankar N. Enterococcus faecalis infection activates phosphatidylinositol 3-kinase 508 signaling to block apoptotic cell death in macrophages. Infect Immun 2014; 82:5132-42.

509 31. Huang XL, Wang YJ, Yan JW, et al. Role of anti-inflammatory cytokines IL-4 and IL-13 in 510 systemic sclerosis. Inflamm Res 2015; 64:151-9.

511 32. Minnone G, De Benedetti F, Bracci-Laudiero L. NGF and Its Receptors in the Regulation of 512 Inflammatory Response. Int J Mol Sci 2017; 18.

513 33. Yano K, Liaw PC, Mullington JM, et al. Vascular endothelial growth factor is an important 514 determinant of sepsis morbidity and mortality. J Exp Med 2006; 203:1447-58.

515 34. Bhattacharya P, Thiruppathi M, Elshabrawy HA, Alharshawi K, Kumar P, Prabhakar BS. GM516 CSF: An immune modulatory cytokine that can suppress autoimmunity. Cytokine 2015; 75:26151771.

518 35. Shimoji K, Yuasa S, Onizuka T, et al. G-CSF promotes the proliferation of developing 519 cardiomyocytes in vivo and in derivation from ESCs and iPSCs. Cell Stem Cell 2010; 6:227-37.

520 36. Martins A, Han J, Kim SO. The multifaceted effects of granulocyte colony-stimulating factor in 521 immunomodulation and potential roles in intestinal immune homeostasis. IUBMB Life 2010; $522 \quad 62: 611-7$ 
523 37. Lee SH, Kwon JY, Kim SY, Jung K, Cho ML. Interferon-gamma regulates inflammatory cell 524 death by targeting necroptosis in experimental autoimmune arthritis. Sci Rep 2017; 7:10133.

525 38. Kothapalli R, Nyland SB, Kusmartseva I, Bailey RD, McKeown TM, Loughran TP, Jr. 526 Constitutive production of proinflammatory cytokines RANTES, MIP-1beta and IL-18 527 characterizes LGL leukemia. Int J Oncol 2005; 26:529-35.

528 39. La Cava A. Leptin in inflammation and autoimmunity. Cytokine 2017; 98:51-8.

529 40. Trinchieri G. Interleukin-12: a proinflammatory cytokine with immunoregulatory functions that 530 bridge innate resistance and antigen-specific adaptive immunity. Annu Rev Immunol 1995; $531 \quad 13: 251-76$

532 41. Singh KV, La Rosa SL, Somarajan SR, Roh JH, Murray BE. The fibronectin-binding protein 533 EfbA contributes to pathogenesis and protects against infective endocarditis caused by 534 Enterococcus faecalis. Infect Immun 2015; 83:4487-94.

535 42. Nallapareddy SR, Qin X, Weinstock GM, Hook M, Murray BE. Enterococcus faecalis adhesin, 536 ace, mediates attachment to extracellular matrix proteins collagen type IV and laminin as well as 537 collagen type I. Infect Immun 2000; 68:5218-24.

538 43. .Nallapareddy SR, Singh KV, Sillanpaa J, et al. Endocarditis and biofilm-associated pili of 539 Enterococcus faecalis. J Clin Invest 2006; 116:2799-807.

540 44. Reardon-Robinson ME, Ton-That H. Disulfide-Bond-Forming Pathways in Gram-Positive $541 \quad$ Bacteria. J Bacteriol 2015; 198:746-54.

542 45. Landeta C, Boyd D, Beckwith J. Disulfide bond formation in prokaryotes. Nat Microbiol 2018; $543 \quad 3: 270-80$

544 46. Daniels R, Mellroth P, Bernsel A, et al. Disulfide bond formation and cysteine exclusion in 545 gram-positive bacteria. J Biol Chem 2010; 285:3300-9. 
546 47. Dutton RJ, Boyd D, Berkmen M, Beckwith J. Bacterial species exhibit diversity in their

547 mechanisms and capacity for protein disulfide bond formation. Proc Natl Acad Sci U S A 2008;

$548 \quad 105: 11933-8$.

549 48. Loh LN, Gao G, Tuomanen El. Dissecting Bacterial Cell Wall Entry and Signaling in Eukaryotic 550 Cells: an Actin-Dependent Pathway Parallels Platelet-Activating Factor Receptor-Mediated $551 \quad$ Endocytosis. MBio 2017; 8.

552 49. Musher DM, Abers MS, Corrales-Medina VF. Acute Infection and Myocardial Infarction. N 553 Engl J Med 2019; 380:171-6.

554 50. Murray BE, Singh KV, Ross RP, Heath JD, Dunny GM, Weinstock GM. Generation of 555 restriction map of Enterococcus faecalis OG1 and investigation of growth requirements and 556 regions encoding biosynthetic function. J Bacteriol 1993; 175:5216-23.

\section{FOOTNOTE PAGE}

\section{Conflict of Interest statement}

560 There are no conflicts of interest.

\section{Funding statement}

562 This work was supported by the National Institute of Dental and Craniofacial Research of the

563 National Institutes of Health under [grant number R01DE027608 to D. A. G.] and [grant number

564 F32DE027580 to A. O. B.]

565 Meetings

566 This work has not been presented.

567 Corresponding author contact information

568 Danielle A. Garsin, Ph.D.

5696431 Fannin St.

570 MSB 1.168 
bioRxiv preprint doi: https://doi.org/10.1101/2020.01.14.906669; this version posted June 5, 2020. The copyright holder for this preprint (which was not certified by peer review) is the author/funder. All rights reserved. No reuse allowed without permission.

\section{Houston, TX 77030}

572 Telephone: (713) 500-5454

573 Fax: (713) 500-5499

574 Email: Danielle.A.Garsin@uth.tmc.edu

575 
Figure 1

A.

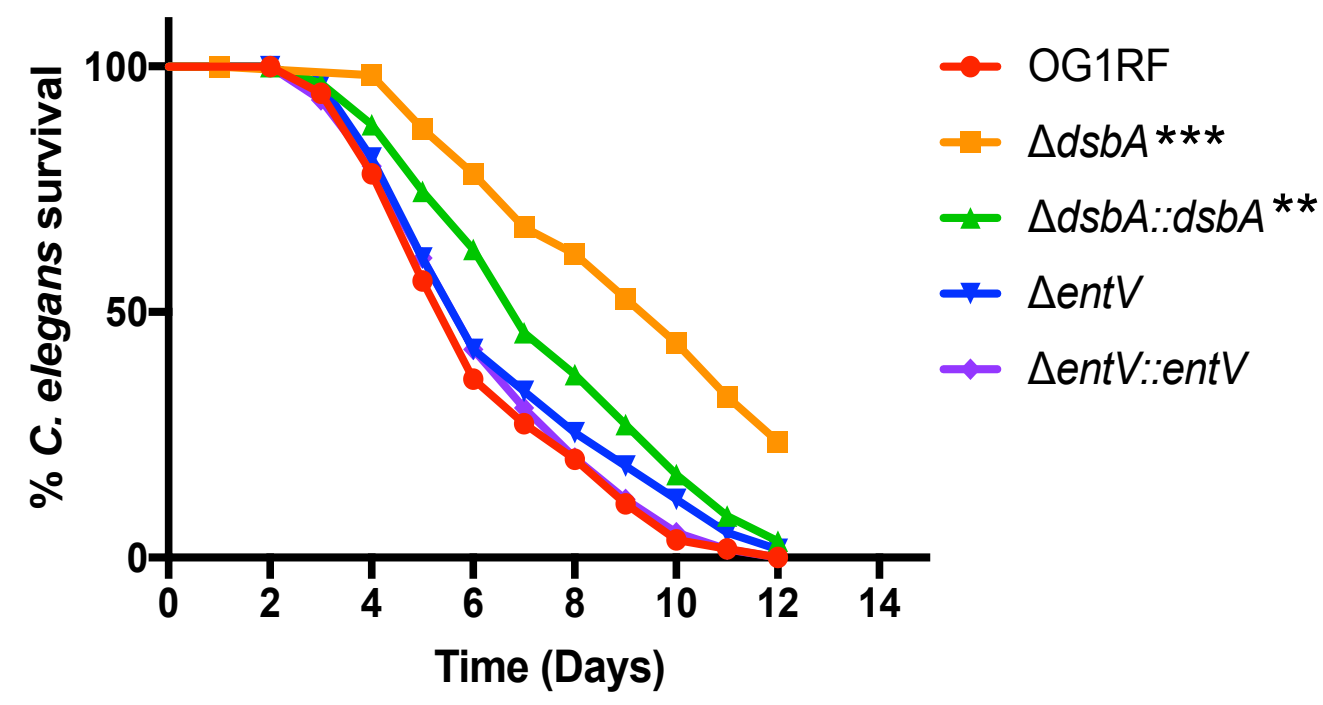

B.

$\Delta d s b A::$

OG1RF $\Delta d s b A d s b A$

$50 \mathrm{kDa}-$

$37 \mathrm{kDa}$

$25 \mathrm{kDa}$

$20 \mathrm{kDa}$

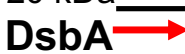

(19.6 kDa) 
Figure 2
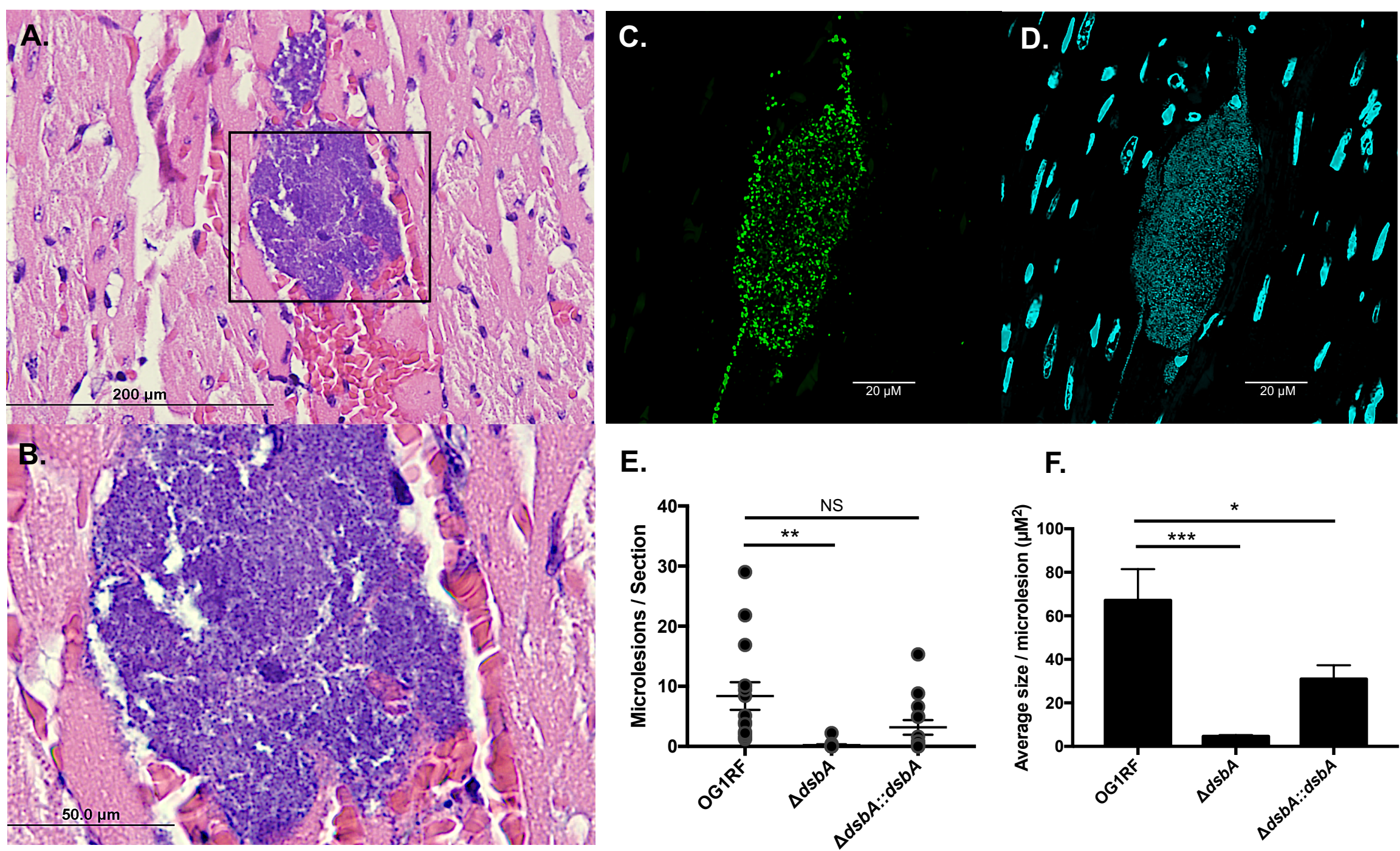

E.

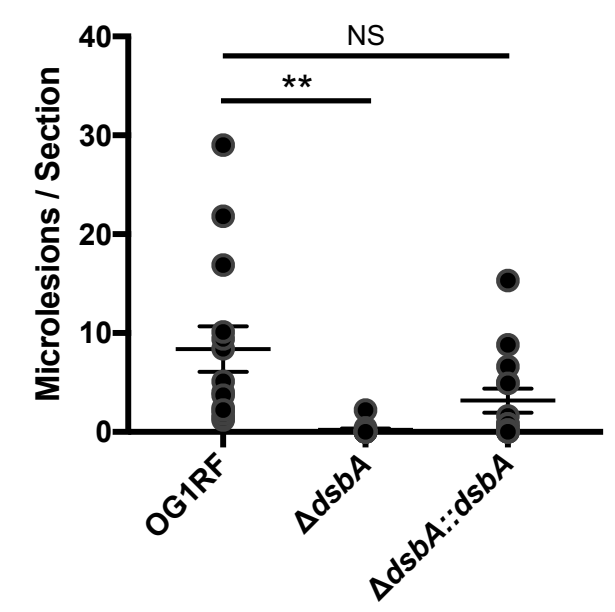

F.

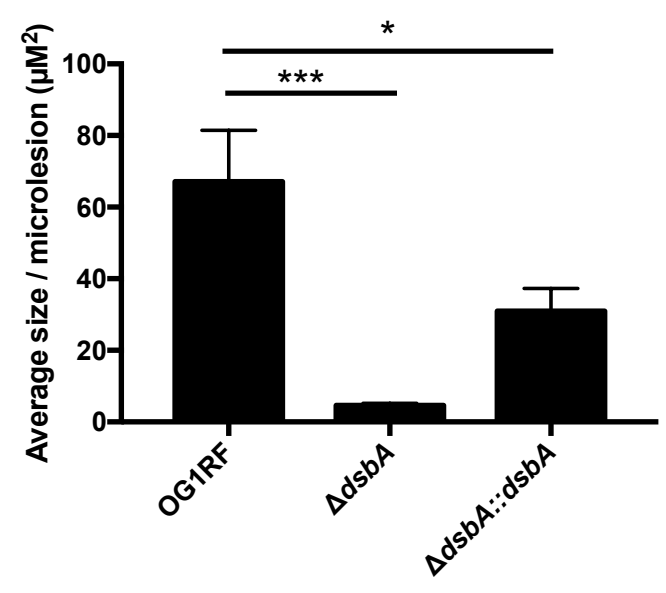


Figure 3

A.

E. faecalis

PARP

Merge

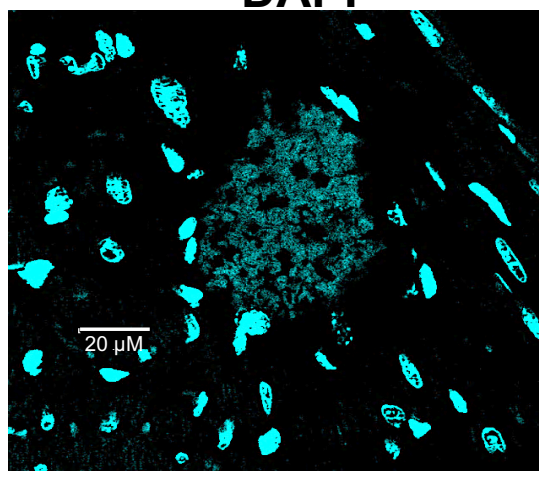

B.

DAPI

$\overline{20 \mu \mathrm{M}}$

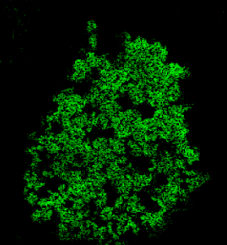

$\frac{1}{4}$

$\overline{20 \mu \mathrm{M}}$

$\overline{20 \mu \mathrm{M}}$
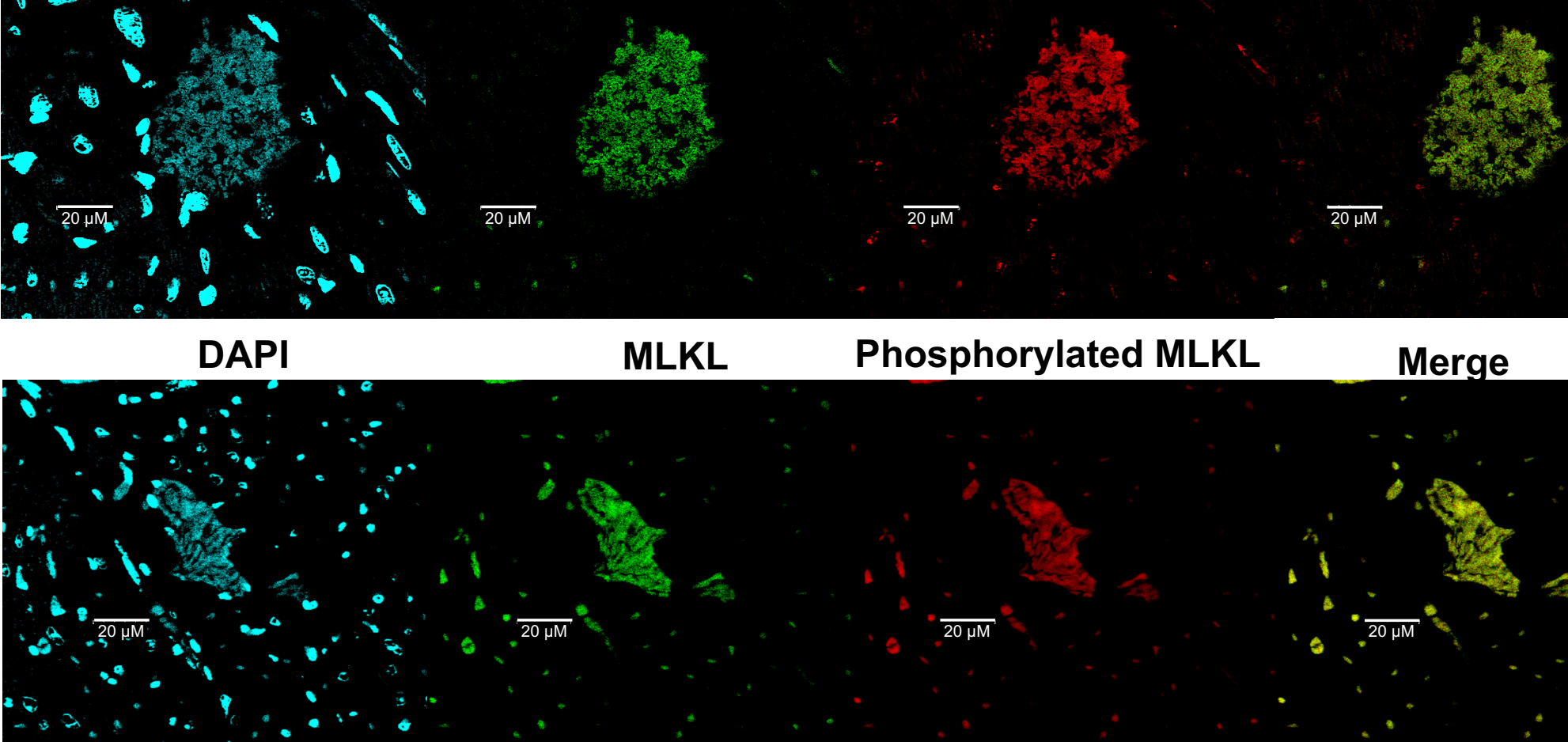

Phosphorylated MLKL

Merge

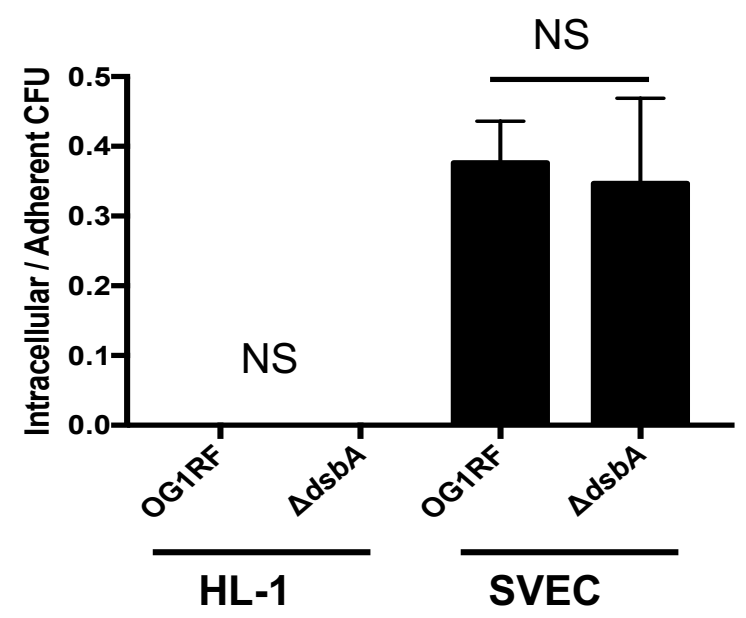

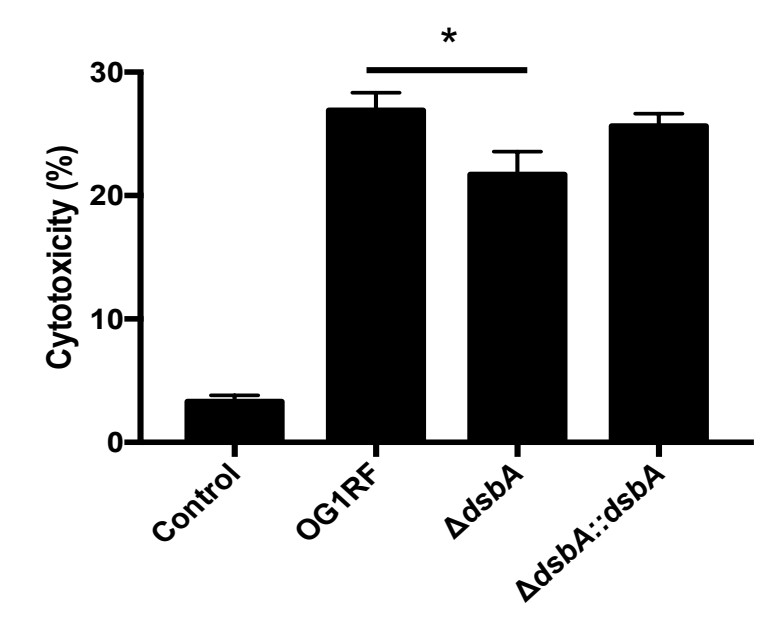

C.
D.

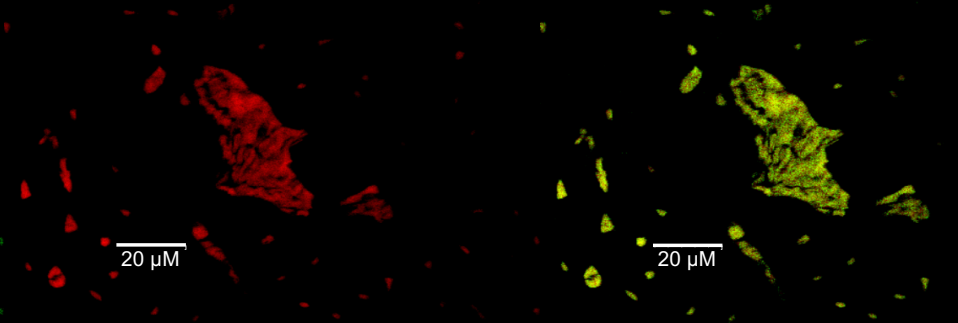


Figure 4

A.

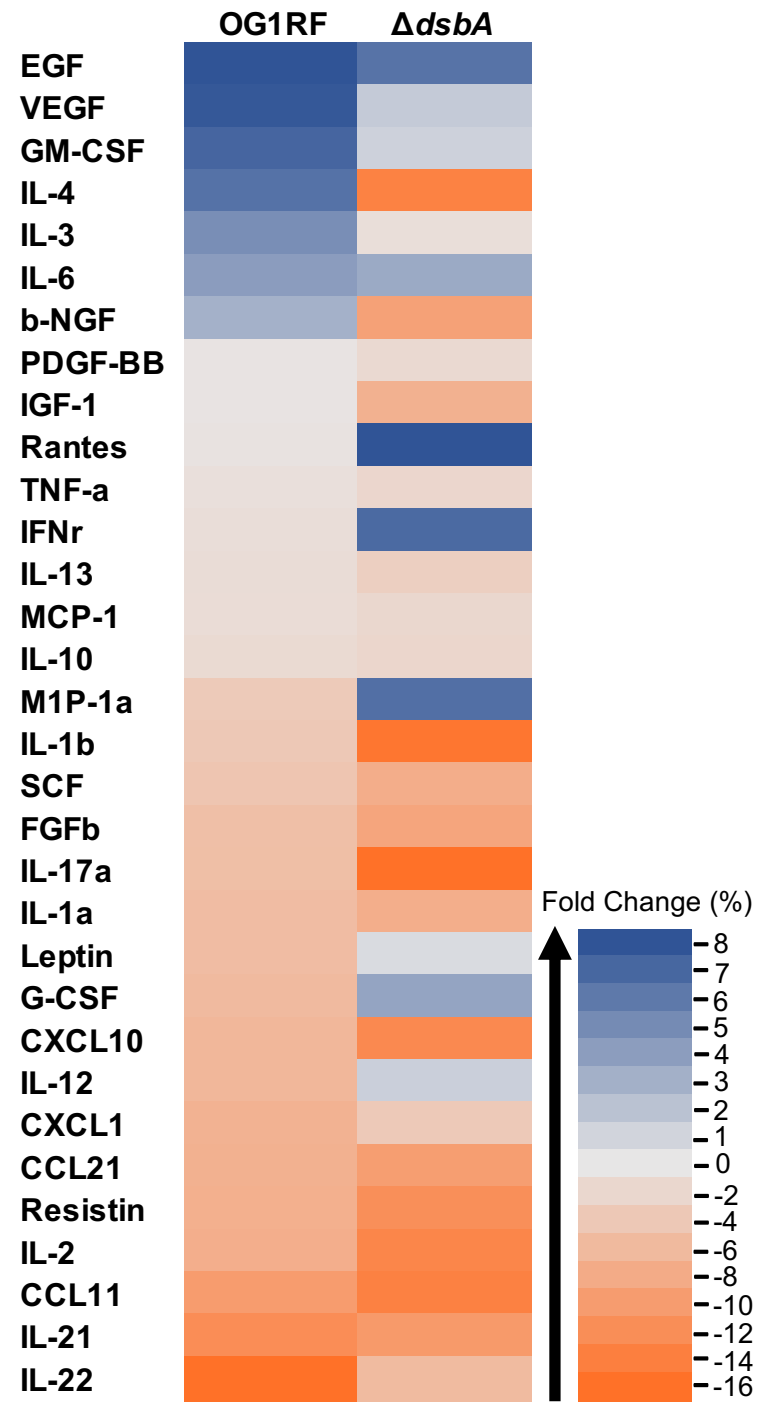

B.

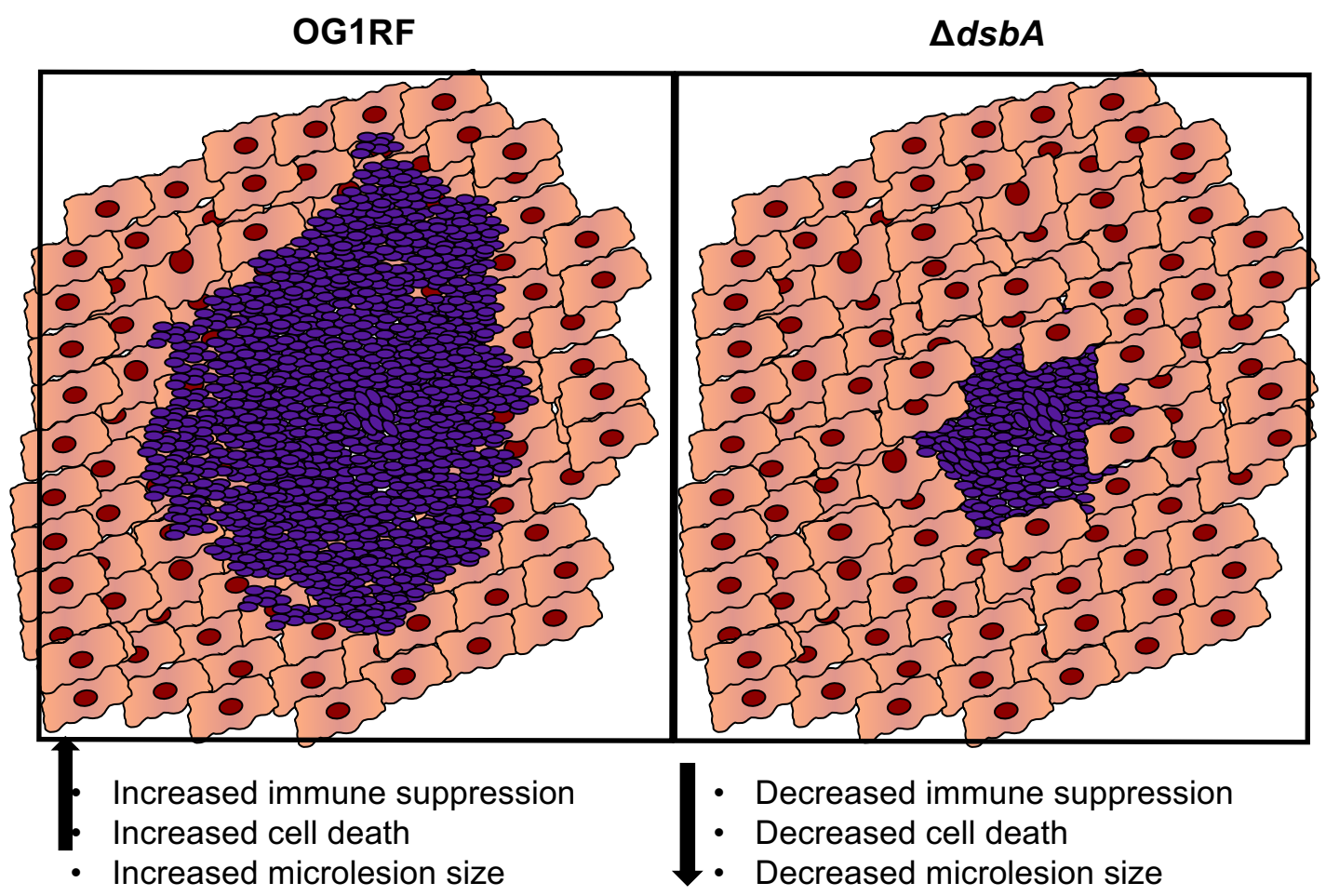

